\title{
Refractive Index and Temperature Sensor Using HC-1550 Infiltrating by Different Liquid Crystal
}

\author{
Suha Mousa Khorsheed Alawsi, Mariam Abdul Jabbar \\ Department of Physics, College Sciences, Al-Nahrian University, Baghdad, Iraq \\ Email: suhaalawsi@gmail.com
}

How to cite this paper: Alawsi, S.M.K. and Jabbar, M.A. (2018) Refractive Index and Temperature Sensor Using HC-1550 Infiltrating by Different Liquid Crystal. Optics and Photonics Journal, 8, 29-39. https://doi.org/10.4236/opj.2018.83004

Received: December 22, 2017

Accepted: March 19, 2018

Published: March 22, 2018

Copyright (c) 2018 by authors and Scientific Research Publishing Inc. This work is licensed under the Creative Commons Attribution International License (CC BY 4.0).

http://creativecommons.org/licenses/by/4.0/

\section{(c) (i) Open Access}

\begin{abstract}
Photonic crystal fiber (PCF) is employed as a refractive index sensor (RIS) for solving a lot of problems in biological, physicochemical, medical, engineering fields and many environmental challenges, where it is used in many industries of food, medicines, chemical materials and material diagnosis. The kind of the PCFs was HC-1550 with three wavelengths of laser source were used; $638 \mathrm{~nm}$, $850 \mathrm{~nm}$, and $1550 \mathrm{~nm}$ that are useful for recording the intended transmitted signal intensity. Therefore, the measured RI was in the range (1.469 - 1.455 $R I C)$ at the temperature range $(36-70)^{\circ} \mathrm{C}$ for the EBBA and it was $(1.621-$ $1.612 \mathrm{RIU}$ ) at the temperature range $(22-42)^{\circ} \mathrm{C}$ for the MBBA. The results showed that the highest RI sensitivity was $96.335 \mathrm{dBm} / \mathrm{RIU}$ for the HC-1550 infiltrated with MBBA using the laser of wavelength $638 \mathrm{~nm}$, Also, the highest temperature sensitivity was $0.2505 \mathrm{dBm} /{ }^{\circ} \mathrm{C}$ for empty $\mathrm{HC}-1550$ using the laser of wavelength $1550 \mathrm{~nm}$.
\end{abstract}

\section{Keywords}

Refractive Index Sensor, Temperature Sensor, Photonic Crystal Fiber, Liquid Crystal

\section{Introduction}

In problems of sensitivity measurements based on refractive index, the RIS uses a PCF which is either immersed in the solution of that materials or infiltrated a specific material inside the PCF, which leads to shifting the wavelength of the transmitted light after the infiltration, then RIS can be used to investigate the purity of the water or used to know the ratio of the drug in the medicines and endlessly applications. The sensitivity test also requires using different types of 
resources, such as: Laser sources, PCF types, and infiltration materials.

Kuhlmey B. T. et al. in 2009 have the study of the ultrasensitive photonic crystal fibers refractive index sensor, where they used the solid core photonic crystal fiber in a directional coupler architecture to introduce the microfluidic refractive index sensor. To use the selective infiltration of any individual hole along the PCF and by this method of precise infiltration they can design the sensor with high sensitivity through the coupling of the mode of the core which is in the adjacent waveguide that fluid-filled which is beyond the modal cutoff. They obtain that the sensitivity was $30,000 \mathrm{~nm} / \mathrm{RIU}$ and the detection limit was $4.6 \times 10^{-7} \mathrm{RIU}$ and for that date this is the highest sensitivity which can be obtained [1]. Bing P. B. et al. in 2012 studied a photonic crystal fiber based on surface Plasmon resonance temperature sensor with liquid core, where Glycerin liquid that has a high RI was filled with the Hollow Core HC-PCF in its central air hole, i.e., the guiding mechanism will be Total Internal Reflection (TIR), which made the transmission bands be broadened. If there is an ambient temperature of the glycerin, it can be detected accurately by the change of the RI of the glycerin with the change in the temperature resolution of the PCF-SPR temperature sensor that was low about $4 \times 10^{-6} \mathrm{RIU}$ [2]. Ana M. R. Pinto and Manuel Lopez-Amo in 2012 had the studied of the HC-PCF refractive index sensor based on modal interference, where they are splicing both ends of HC-PCF with a single mode fiber SMF by fusion splicing, there is an air collapse, the modal interference was formed by exciting and recombining core and cladding modes. They used the sugar solution to make the shifting in the wavelength in the interference spectra in the HC-PCF. The RI range was (1.333 - 1.3775) RIU. The resulted resolution and the sensitivity were $5.53 \times 10^{-4} \mathrm{RIU}$ and $36.184 \mathrm{~nm} / \mathrm{RIU}$, respectively and the temperature sensitivity about $19 \mathrm{pm} /{ }^{\circ} \mathrm{C}$ with the using temperature range $(35.5-60.5)^{\circ} \mathrm{C}$ and it can used for chemical, biological and biochemical applications [3].

Abdulhadi A. H. and Al-janabi A. H. in 2012 used the solid core SC-PCF based on Mach-Zehnder modal interferometer for temperature and refractive index sensing, where they used a short length of the PCF splicing in both side with conventional SMF by fusion splicing and he used the laser (1525 - 1610) $\mathrm{nm}$. The interference spectrum is unchanged as indicated at temperature range was $(25-60)^{\circ} \mathrm{C}$, while the heads of the sensor could realize very high temperature up to $550^{\circ} \mathrm{C}$. Dimethyl Sulfoxide (DMSO) was the liquid which was infiltration between the PCF piece and the tube of silica. The temperature sensitivity of the temperature was very high than the previous published work on the PCF sensors of temperature. Also Dimethyl form amide (DMF) liquid was tested and it shows a temperature sensitivity reached to $0.7 \mathrm{~nm} /{ }^{\circ} \mathrm{C}$. Yee O. S. in 2014 has the study of the refractive index sensor based on the hollow-core photonic crystal fiber, where he did this using the technique of modal interference. He obtained two kinds of sensor through this research. The first one was the refractive index sensor and the second one was the temperature sensor where he used to design the refractive index sensor. The arc fusion splicing to splice the PCF with 
the SMF, sure there is an air hole collapse region and the HC-PCF that used to do this splicing was short less than $1 \mathrm{~mm}$. He has to monitor experimentally the wavelength that appears in the transmission spectra. Besides, through the RIS he could obtain the temperature sensor. The results demonstrate for refractive index $n$ range (1.333 to 1.3775 ) that the measurement resolution was $5.53 \times 10^{-4}$ RIU and the sensor sensitivity was $36.184 \mathrm{~nm} / \mathrm{RIU}$ and the temperature range was $(35.5-60.5)^{\circ} \mathrm{C}$ and the temperature sensor sensitivity was $19 \mathrm{pm} /{ }^{\circ} \mathrm{C}[4]$.

The implementation is starting by splicing the PCF between two Conventional Optical Fiber (COF) to align the light signal through the PCF and received it by a spectrometer to The implementation is starting by splicing the PCF between two Conventional Optical Fiber (COF) to align the light signal through the PCF and received it by a spectrometer to records the results. The gained experience enable to make the Mechanical Splicing (MS) instead of the Fusion Splicing (FS) that used in most researchers, MS is considering as a cold splicing and prevents the holes collapse as a converse to the FS which provides a huge temperature reaches to $600^{\circ} \mathrm{C}$ that destroy the infiltrated materials and loss it natural properties and make distorted results although its low loss in the signal like evaporation of the water. The used infiltration method was carrying out using the standard medical syringe which was gluing with the PCF using some kinds of the glue, which is pumping by human hand to push the material inside the PCF. This manual infiltration method pushes the materials for more than two centimeters inside the PCF, which is more reliable method than the conventional capillary method that cannot push the material a few centimeters only.

Two types of infiltration materials are used. They are nematic Liquid Crystals (LCs): EBBA and MBBA. These materials have special properties are useful in the RIS tests; the temperature range is $(36-80)^{\circ} \mathrm{C}$ for EBBA and $(22-42)^{\circ} \mathrm{C}$ for MBBA. An appropriate oven has been designed and used throughout the implementation to vary the temperature of the PCF before and after the infiltration within the mentioned allowed ranges. Variation of the temperature affects the RI of infiltrated materials that can be measured to indicate the performance of the proposed RIS. To ensure the valid path of the implementation, the chemical properties of the used materials was tested using the Fourier Transform Infrared Spectroscopy (FTIS), the optical properties were tested using the refractometer, while the microstructure was tested using the Scanning Electron Micrograph (SEM) and Energy Dispersion Spectroscopy (EDS).

\section{PCF Based Temperature and Refractive Index Sensors}

The effect of temperature variation impacts the LC density, which is directly proportional to the refractive index. When the temperature rises, it lead to a decrease in the LC density this is due to high temperature make the LC stretched and loss the strength of attraction between molecules and thus get bigger distances between particles and increases the internal molecular movement [5]. The measurements are recording from the oven regulator and Optical spectrum analyzer (OSA). The oven regulator controls the degree of temperature in the oven 
that is same that of the PCF inside the oven. For different amounts of temperatures within an allowed range, the OSA records the transmission power of the PCF measured in $\mathrm{dBm}$. The graph of the recorded transmission power as a function of temperature indicates the behavior of the PCF against temperature. The sensitivity of the sensor will be determined by estimating the slope of transmission behavior with temperature variations [6].

$\mathrm{RI}$ is one of the fundamental distinguishable optical properties for each material, where it considered as one of the phytochemical properties of the materials due to it specify the effects of the electromagnetic waves on the material. RI dependents on the density and the wavelength, where the refractive index of some material is the ratio between the speed of light in the vacuum and the speed of light in that material as indicated in the following relationship [6]:

$$
n=\frac{c}{v}
$$

where the material refractive index is $n$, the speed of the light in the material is $V$, and $c$ is the speed of light in the vacuum. Therefore, the index of refraction makes to decrease the speed of light inside the medium according to the following relationship:

$$
\frac{n_{0}}{\lambda_{0}}=\frac{n_{1}}{\lambda_{1}}
$$

where, $n_{0}$ is the refractive index of the material before the changing of the temperature, $n_{1}$ is the refractive index of the material after the changing of the temperature, $\lambda_{0}$ is the wavelength of the material before changing the temperature, and $\lambda_{1}$ is the wavelength of the materials after changing the temperature [7]. The refractive index measured by refractometer via two methods; the change the temperature or by specifying the Brix degree i.e., by changing the material concentration. It depends on the density and temperature, where the refractive index decreases when the temperature increase i.e., the density increase. Also, the refractive index can be specified the dispersive power of the prism and focusing the power of lenses or can know the purity some materials like the water by specifying the refractive index by the changing the concentration of it [8]. The experimental setup of the refractive index sensor is same as that of the temperature sensor shown in Figure 1. The measurement in such case is recorded between the variations of the transmission power using the OSA to the variation of the refractive index that occurred due to the temperature variations. Since the relation between the refractive index and temperature is inversely proportional, the graph that indicates the behavior of the transmission as a function of refractive index is the inverse of the behavior of the transmission as a function of temperature [6] [7]. Also, the refractive index sensor can act by changing the wavelength of the laser source that proper to the PCF and then be recording the resulted wavelengths according to "Equation (2)" where the refractive index of the materials is computing when the two wavelengths before and after the infiltration are known via spectrometer, and the first RI of the air is also known. 


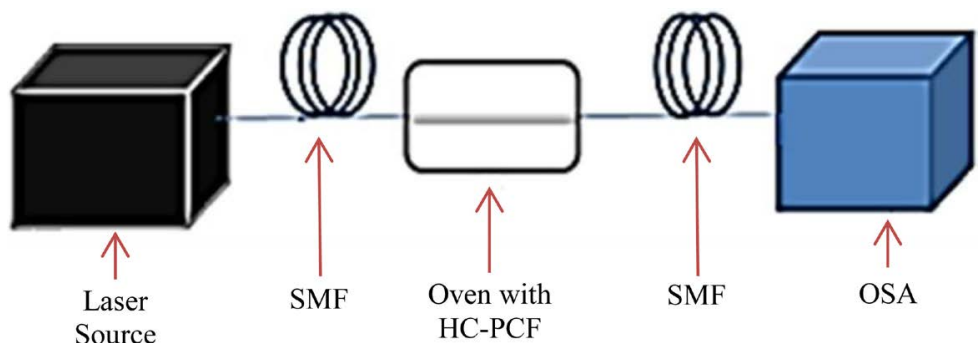

Figure 1. Requirements of refractive index sensor and setup.

The OSA providing the monitoring and measurement of some information about the transmitted light through the PCF in addition to the wavelength like the transmitted intensity in $\mathrm{dB}$ and by using "Equation (2)" the transmitted intensity in $\mathrm{dBm}$ is obtained. Then, the sensitivity of the refractive index sensor is equivalent to the slope of the graph that indicates the behavior of transmission power measured by $\mathrm{dBm}$ as a function of the refractive index [6].

\section{Design Requirements and Setup}

The analytical study shows that there are different components were required to implement the proposed design of RIS. Such components were related to the devices, material, and tools that needed to establish the present work. Figure 1 shows the setup of the proposed design, the main components are explained with more details in the following:

1) Laser source: it used to provide the light source to the experiment.

2) Single mode fiber (SMF): it used to guide the laser light inside the measurement zone.

3) Connector: it used to connect the SMF with the HC-PCF that found in the measurement zone.

4) HC-PCF: it used to guide the laser light inside the measurement zone.

5) LC materials: they used to fill the HC-PCF at different test situations.

6) Oven: it used for providing a specific temperature for each test situation around the HC-PCF.

7) OSA: it used to measure the output power of the laser light.

\section{RIS Experiment Implementation}

This paper was consists of many steps such as oven design, LCs properties tests, laser insertion through the PCF and received it by the OSA, and LCs infiltration through the PCF, where the chosen PCFs were the hollow core (HC-PCF) such as HC-1550, with an available proper laser wavelengths were $638 \mathrm{~nm}, 850 \mathrm{~nm}$, and $1550 \mathrm{~nm}$, the using LCs were the EBBA and MBBA and testing devices for their properties were the FTIR to test their chemical properties such as their purity, diagnosis the material itself, or checkup their performance, the optical properties were checkup via refractometer, where this device provides the refractive index for different temperature, and the microstructure was tests by EDS and the topography pictured by SEM, the oven made of aluminum with temper- 
ature controller provided by thermocouple and regulator, the skills of insertion of the laser signal precisely inside the PCF and received it by the spectrometer with more accuracy results, where the using spectrometers were the (OSA) and the near infrared spectrum analyzer and these spectrometers have connectors proper to that used in the optical fiber $(\mathrm{OF})$ and the using lasers were tunnel lasers that proper to the OF with low loss in the signal light, this process done by preparing the surface of the PCF and the COF ends by using stripper, cleaner liquid, or cleaver and by mechanical splicing, the PCF spliced between two conventional $\mathrm{OF}$ that of single mode fiber (SMF) and multimode fiber (MMF) kinds to increasing the sensing process for some cases like the HC-800 instead of the MMF with MMF or SMF with the SMF, infiltration of the liquid crystal inside the PCF done by using the standard medical syringe by gluing it with the PCF using a glue and the LC infiltrated by the pressure, this processes were done for each PCF with each LC and by specifying the wavelength shift of the peak after the infiltration of the LCs according to the "Equation (2)" and calculate the sensitivity for each case and specify which one of these cases was the more sensitive than the other.

\section{Results and Discussion}

Practically, Figures 2-12 show that the transmission appeared inversely proportional to the temperature, where it is directly proportional to the refractive index. It shown that the behavior of the transmissions with respect to temperature indicates that the transmitted signal decrease with increasing the temperature of the PCF, this is due to the collisions between the molecules of the silica and the light signal that will losses the signal part of its motion power. In such situation, the refractive index is also decreases due to its inverse proportionality with the temperature. The diagrams in the cases of the empty PCF between the refractive index and the transmitted signal were eliminated since the refractive index of the air is constant according to the Equation (1), where the $\mathrm{n}$ represent the refractive index of the medium, $\mathrm{c}$ is the velocity of light in vacuum and $\mathrm{v}$ is the speed of the light in that medium. When the velocity in the medium inside the PCF was the same as $c$, then the refractive index of the air was constant equal to 1 . Through the experimental tests, it is found that the laser with wavelength $638 \mathrm{~nm}$ was appeared in all the cases of the splicing state with high intensity easily as compared with the other laser wavelength, the laser $850 \mathrm{~nm}$ was more than the laser 1550 $\mathrm{nm}$ and in all cases, while laser $1550 \mathrm{~nm}$ was not appeared in the cases HC-1550 except for the cases of air because of the absorption of the materials to the wavelength $1550 \mathrm{~nm}$ i.e., the wavelength affected by the media of the PCF. The wavelength that used in the refractometer is directly affecting the results, White light such as light emitting diode LED used in the refractometer tests that made the results contain all the wavelengths of the tested laser. It is noticeable that the case in which passes the laser come from MMF directed to SMF is different from that when the laser comes from SMF toward MMF. Moreover, there were some errors are found in the theoretical measurements such as: attenuation, dispersion, 


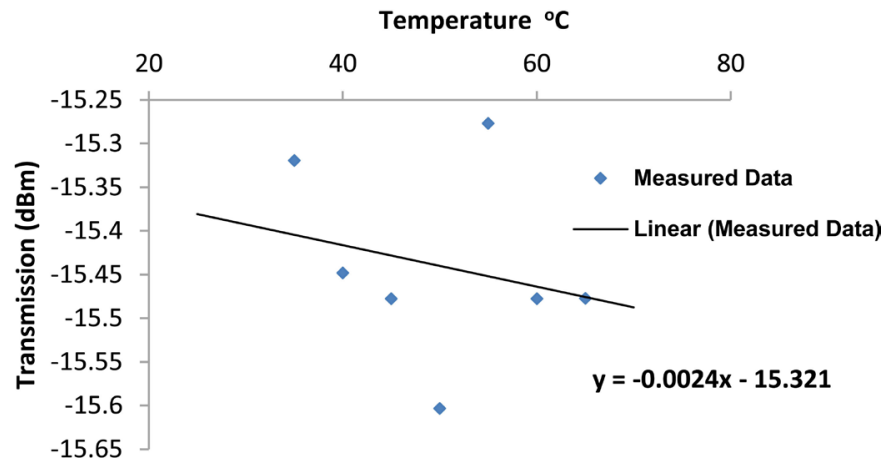

Figure 2. Transmission with different temperature for HC-1550 at wavelengths $638 \mathrm{~nm}$.

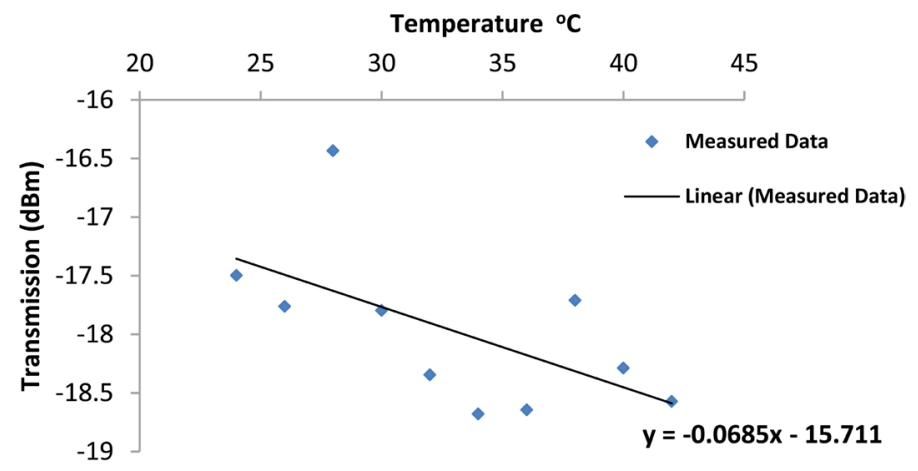

Figure 3. Transmission with different temperature for empty HC-1550 at $850 \mathrm{~nm}$.

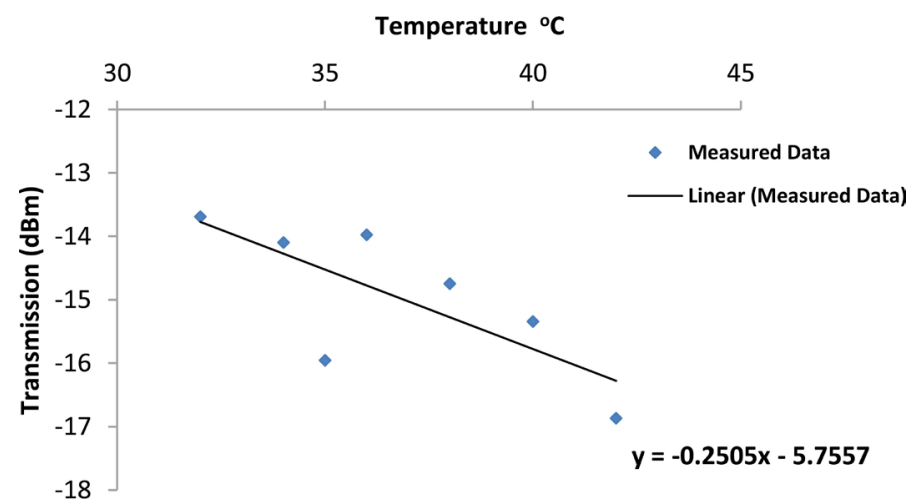

Figure 4. Transmission with different temperature for empty HC-1550 at $1550 \mathrm{~nm}$.

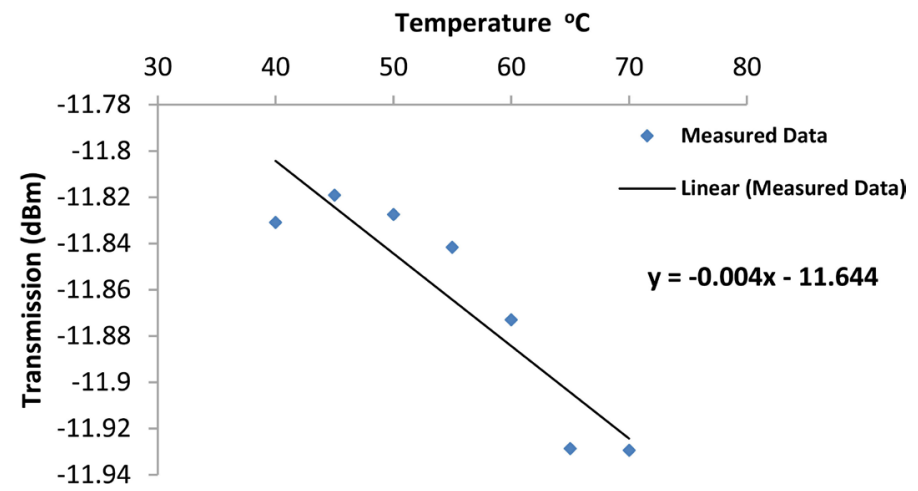

Figure 5. Transmission with different temperature for HC-1550 infiltrated with EBBA at wavelengths $638 \mathrm{~nm}$. 


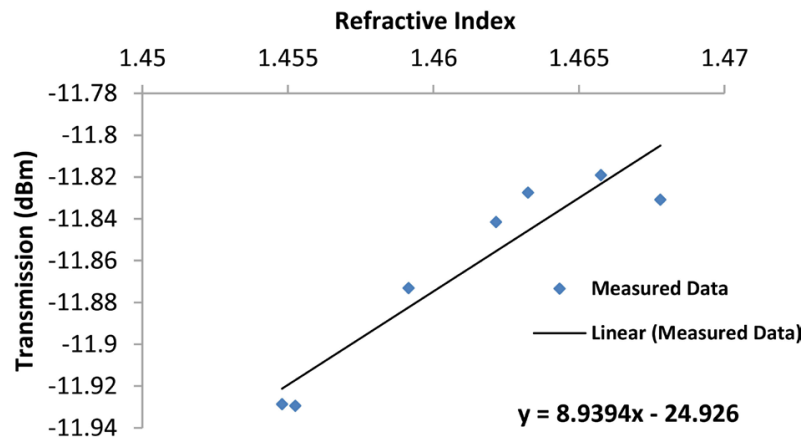

Figure 6. Transmission with different refractive index for HC-1550 infiltrated with EBBA at wavelengths $638 \mathrm{~nm}$.

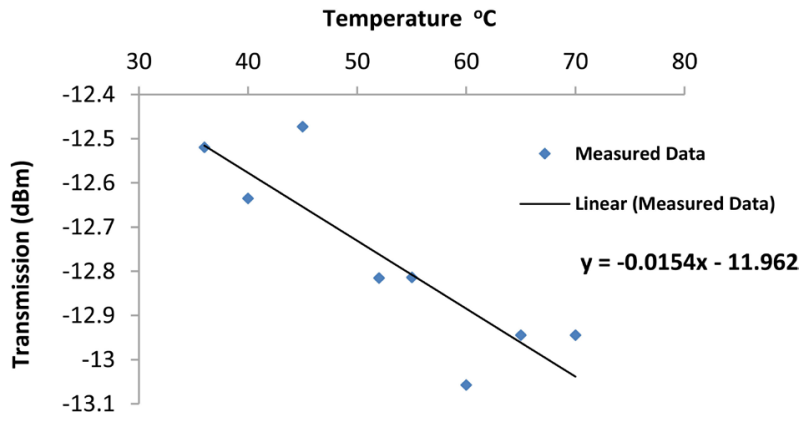

Figure 7. Transmission with different temperature for $\mathrm{HC}-1550$ infiltrated with EBBA at $850 \mathrm{~nm}$.

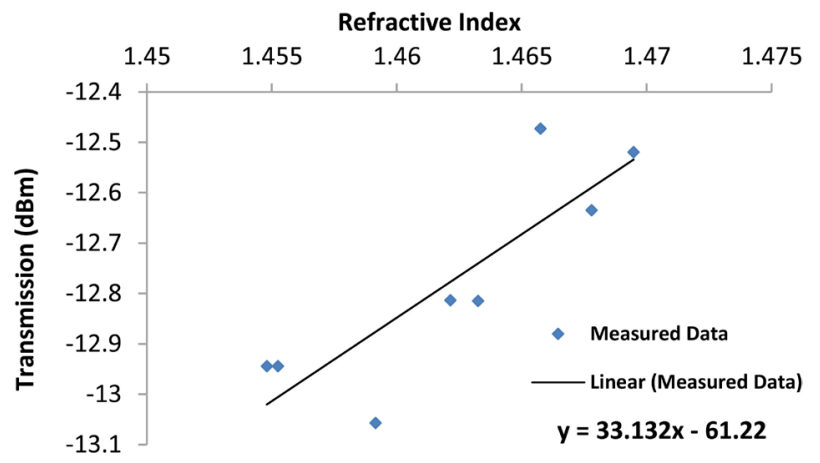

Figure 8. Transmission with different refractive index for HC-1550 infiltrated with EBBA at $850 \mathrm{~nm}$.

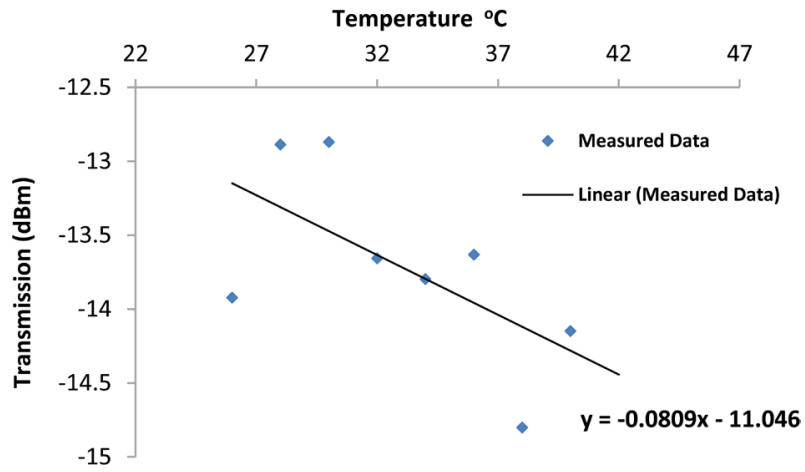

Figure 9. Transmission with different temperature of HC-1550 infiltrated with MBBA at wavelengths $638 \mathrm{~nm}$. 


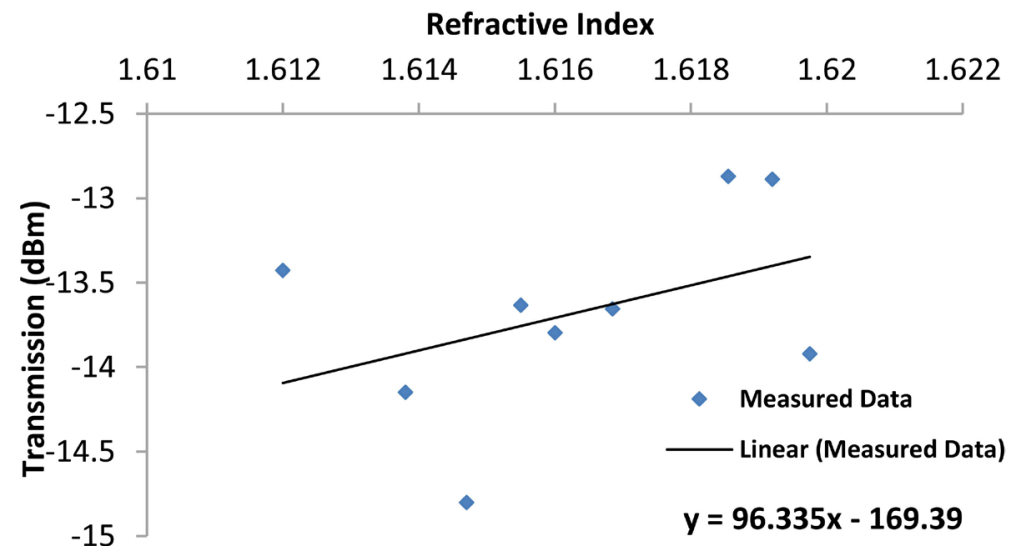

Figure 10. Transmission with different refractive index for HC-1550 infiltrated with MBBA at wavelengths $638 \mathrm{~nm}$.

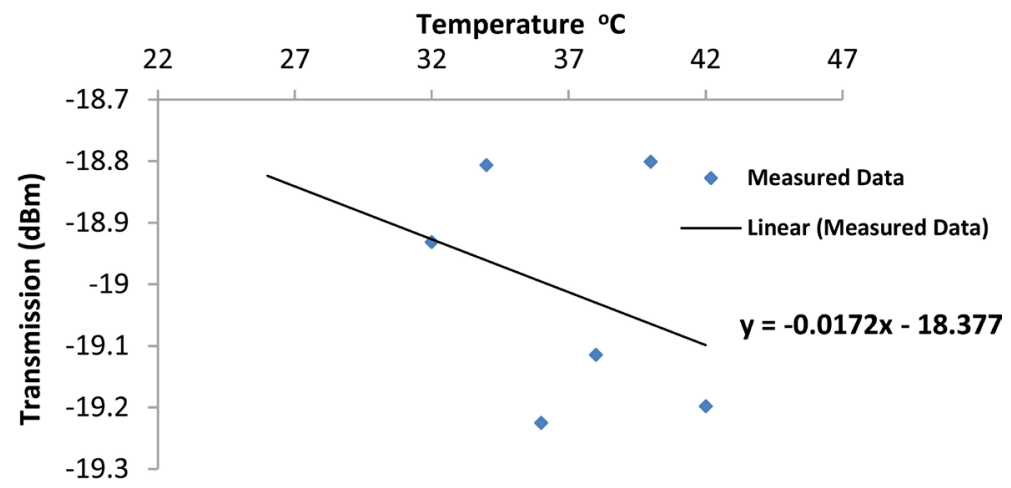

Figure 11. Transmission with different temperature for HC-1550 infiltrated with MBBA at $850 \mathrm{~nm}$.

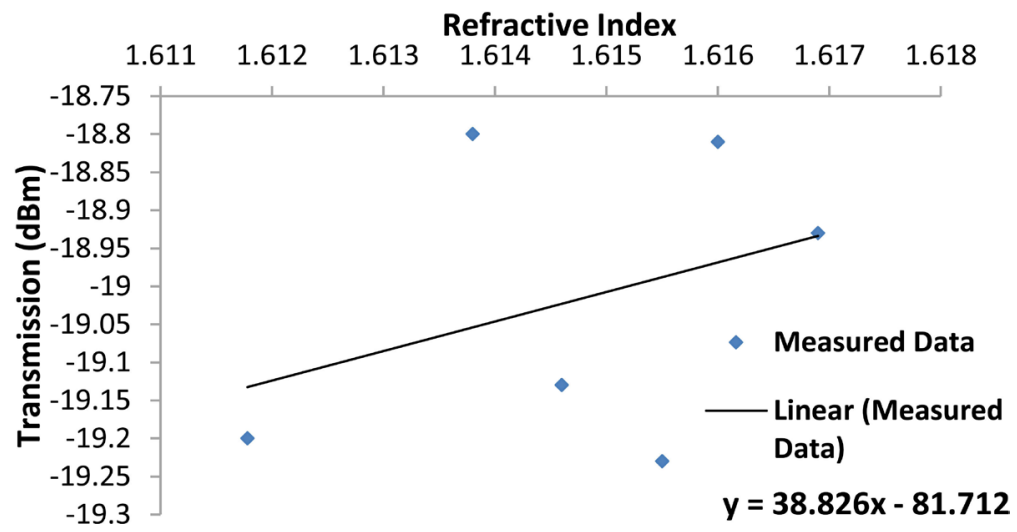

Figure 12. Transmission with different refractive index for HC-1550 infiltrated with MBBA at $850 \mathrm{~nm}$.

splice loss and so on. The practical test shows that the MS loss does not greatly affect the accuracy of the results, which almost remains more accurate than the FS. The proportionality between the wavelengths of the source with the COF and with the operating wavelength of the PCF affected the accuracy of the results, which indicates that high intensity of the PCF signal makes the susceptibility of the external environmental effects to be small. 


\section{Conclusions}

The most important conclusions that indicated throughout the design and implementation of the present work are given in the following:

1) The mechanical splicing between PCF and COF for inserting and receiving the laser signal is more suitable than the fusion splicing, where the FS provides temperature more than $600^{\circ} \mathrm{C}$ to the spliced ends of the spliced PCFs that leads to damage of the infiltrated materials. It implies that the results were wrong although the low loss in the output signal uses FS.

2) The infiltration using the medical syringe and the pressure by the hand was better than the capillary method since the capillary method infiltrated the PCF for a few centimeters while infiltration by human hand using medical syringe infiltrated more length of PCF, which leads to increasing the sensitivity of the PCF to the parameters of the infiltrated materials.

3) The increase of the temperature is leading to decrease of the intensity of the output signal that transmitted in the PCF.

4) The laser of $\lambda=638 \mathrm{~nm}$ was appropriate to be used with the used three types of PCF; the sensitivity is maximum when the $\lambda$ was near the operating $\lambda$.

5) The MS enables to splice the PCF at different cases that may be employed to increase the Sensitivity: like the splicing of MMF-PCF-MMF that is suitable for laser of $\lambda=850 \mathrm{~nm}$, which is a case that cannot be achieved by FS.

6) The highest RI sensitivity was $96.335 \mathrm{dBm} / \mathrm{RIU}$ for the HC-1550 infiltrated with the MBBA using the laser of wavelength $638 \mathrm{~nm}$. While the highest temperature sensitivity was $0.2505 \mathrm{dBm} /{ }^{\circ} \mathrm{C}$ for empty $\mathrm{HC}-1550$ using the laser with wavelength $1550 \mathrm{~nm}$. The intensity loss in the output signal affected the measured sensitivity values.

\section{References}

[1] Kuhlmey, B.T., et al. (2009) Ultrasensitive Photonic Crystal Fiber Refractive Index Sensor, University of Sydney. Optics Letters, 34, 322-324.

https://doi.org/10.1364/OL.34.000322

[2] Bing, P.B., et al. (2012) Fiber Based on Surface Plasmon Resonance Temperature Sensor with Liquid Core. Modern Physics Letters, 26, 50-62. https://doi.org/10.1142/S0217984912500820

[3] Baharin, N.F. et al. (2016) Hollow-Core Photonic Crystal Fiber Refractive Index Sensor Based on Modal Interference. Journal of Engineering and Applied Sciences, 11, 5702-5706.

[4] Al-Janabi, A. and Abdulhadi, A.H. (2010) Photonic Crystal Fiber Based on Mach-Zehnder Modal Interferometer for Sensing Applications. University of Baghdad, Institute of Laser for Postgraduate Studies, Baghdad.

[5] Rindorf, L. and Bang, O. (2013) Sensitivity of Photonic Crystal Fiber Grating Sensors: Biosensing, Refractive Index, Strain, and Temperature Sensing. COM.DTU, Department of Communications, Optics and Materials, Technical University of Denmark, DK-2800 Kgs. Lyngby, Optical Society of America, Denmark.

[6] Shaik, S. and Desai, A., (2014) Temperature Sensor By Using Selectively Filled Photonic Crystal Fiber Sagnac Interferometer, Dept. of Electronics and Telecommuni- 
cation, DJSCOE, Mumbai, India. International Journal of Interdisciplinary and Multidisciplinary Studies (IJIMS), 1, 103-106.

[7] Jorge, P.A.S. (2014) Fiber Optic-Based Refractive Index Sensing at INESC Porto. Sensors, 12, 8371-8389. https://doi.org/10.3390/s120608371

[8] Yee, O.S., (2014) Refractive Index Sensor Bases on Hollow-Core Photonic Crystal Fiber. Faculty of Electrical Engineering, Universiti Teknologi Malaysia, Skudai. 